\title{
Three-Dimensional Evaluation of Pharyngeal Morphology in Bimaxillary Surgery with and without Horseshoe Osteotomy in Skeletal Class III Cases
}

\author{
Naoyuki Yoshino ${ }^{1)}$, Yasushi Nishii ${ }^{1)}$, Takashi Kamio ${ }^{2)}$, Takashi Takaki ${ }^{2)}$, \\ Takahiko Shibahara ${ }^{2)}$ and Kenji Sueishi ${ }^{1)}$ \\ ${ }^{1)}$ Department of Orthodontics, Tokyo Dental College, \\ 1-2-2 Masago, Mihama-ku, Chiba 261-8502, Japan \\ ${ }^{2)}$ Department of Oral and Maxillofacial Surgery, Tokyo Dental College, \\ 1-2-2 Masago, Mihama-ku, Chiba 261-8502, Japan
}

Received 22 February, 2021/Accepted for publication 14 June, 2021

Published Online in J-STAGE 15 November, 2021

\begin{abstract}
Repositioning of the jaw in orthognathic treatment generates changes in the soft tissues of the maxillofacial region, with consequent changes in the airway. The purpose of this study was to determine how type of orthognathic surgical procedure affected the 3-dimensional morphology of the upper airway. Forty patients were divided into the following 2 groups according to the type of surgical procedure used: a horseshoe osteotomy (HS) group (20 patients, comprising 11 men and 9 women; average age $24.3 \pm 4.5$ years) who underwent bimaxillary surgery; and a LeFort I osteotomy (LF) group (20 patients, comprising 8 men and 12 women; average age 22.5 \pm 4.6 years) who also underwent bimaxillary surgery. Cephalometric measurements were taken and 3-dimensional pharyngeal morphology evaluated in each group. The amounts of maxilla rotation, posterior maxilla impaction, and mandibular setback all revealed a significantly larger value in the HS group. Evaluation of pharyngeal volume revealed a significant decrease in the upper pharyngeal segment in the LF group. A significant decrease in the lower pharyngeal segment was observed in both groups. Differences were noted in postoperative pharyngeal morphology between the two groups. The results of this study suggest that HS has less effect on the upper pharyngeal segment, regardless of the amount of posterior maxilla impaction.
\end{abstract}

Key words: Orthognathic surgery - Airway - Morphology -

Horseshoe osteotomy - 3D analysis

This paper was originally a thesis submitted to the Graduate School of Tokyo Dental College on February $5^{\text {th }}, 2019$ by Naoyuki Yoshino. 


\section{Introduction}

Orthognathic surgery not only decreases skeletal discrepancies in skeletal Class III patients, but also greatly improves facial esthetics. Repositioning of the jaw generates changes in the soft tissues of the maxillofacial region, which simultaneously causes changes in the airway ${ }^{2,6)}$. The role of orthognathic surgery in triggering obstructive sleep apnea (OSA) has recently drawn much attention $^{7,8,24,26)}$. In cases with severe skeletal discrepancies, bimaxillary surgery with LeFort I osteotomy (LF) along with sagittal split ramus osteotomy (SSRO) is said to be preferable in terms of both improvement of facial esthetics and stability compared to single-jaw surgery ${ }^{3)}$.

Taking potential change in the airway into consideration, maxillary advancement may be selected in the treatment plan for bimaxillary surgery in order to lessen the amount of mandibular setback. This results in a decrease in improvement of the anteroposterior chin position ${ }^{10)}$, however, making improvement of facial esthetics difficult. Meanwhile, treatment plans aimed at increasing the amount of mandibular setback involve impaction of the posterior maxilla, creating a clockwise rotation of the occlusal plane. A relationship has been noted between postoperative decrease in pharyngeal volume and positional change in the soft palate following impaction of the posterior maxilla when such skeletal movements arise due to orthognathic surgery ${ }^{11}$.

In cases where large amounts of posterior maxilla impaction are needed, LF with horseshoe osteotomy (HS) may be selected. Its advantages include substantial impaction of the maxilla and a low risk of damage to blood vessels and nasal cavities ${ }^{9}$. While a number of studies have investigated the effects of LF on pharyngeal morphology $y^{4,6,10,11,13,15,19,20,22)}$, to our knowledge, no 3-dimensional (3D) analyses of Class III cases involving HS have been performed to date. The aim of this study was to determine how type of orthognathic surgical procedure used affected the 3D morphology of the upper airway.

\section{Materials and Methods}

\section{Patient selection}

A total of 40 patients were enrolled in the study. Skeletal mandibular prognathism had been diagnosed in all these patients at Tokyo Dental College Chiba Hospital between 2000 and 2014, and all had undergone bimaxillary surgery with a planned posterior maxilla impaction of more than $2.0 \mathrm{~mm}$. Cases of severe asymmetry (defined as those where horizontal deviation of the Menton from the facial centric line exceeded $3.0 \mathrm{~mm}$ ), congenital cases such as cleft palate and lip, and cases with past histories of OSA were excluded. The patients were divided into the following 2 groups according to the type of surgery performed: an HS group, comprising 20 patients (11 men and 9 women; average age $24.3 \pm 4.5$ years) who underwent bimaxillary surgery involving LF with HS and SSRO; and an LF group, comprising 20 patients ( 8 men and 12 women; average age, $22.5 \pm 4.6$ years) who underwent bimaxillary surgery involving LF and SSRO. As a retrospective study, lateral cephalograms and multi detector-row computed tomographs (CT) were obtained at 1 month preoperatively (T0) and at 1 month postoperatively (T1). Maxillofacial morphology and the amount of surgical movement were measured at each time point based on the lateral cephalograms, together with pharyngeal volume and pharyngeal morphology based on the CT data. The protocol of this study was approved by the Ethics Committee of Tokyo Dental College (approval number: $753)$.

\section{Measurement methods}

1) Measurements of morphology using lateral cephalograms

Maxillofacial morphology and surgical changes were evaluated based on lateral cephalograms obtained at T0 and T1.

(1) Evaluation of maxillofacial morphology

The evaluated angular measurements comprised the SNA, SNB, mandibular plane angle (MP), and occlusal plane angle (OP), as shown in Fig. 1. Measurements of distance 


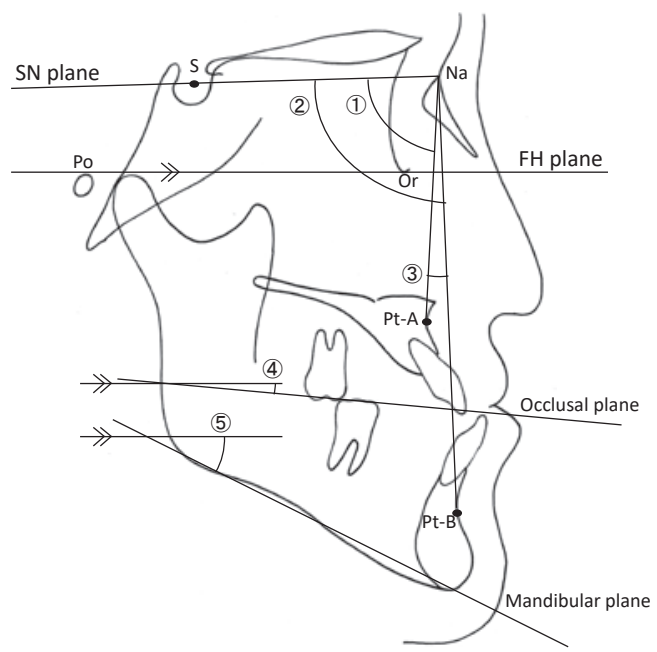

Fig. 1 Angular measurements used in cephalometric analysis

(1) SNA (2) SNB (3) ANB (4) Occlusal plane angle (OP)

(5) Mandibular plane angle (MP)

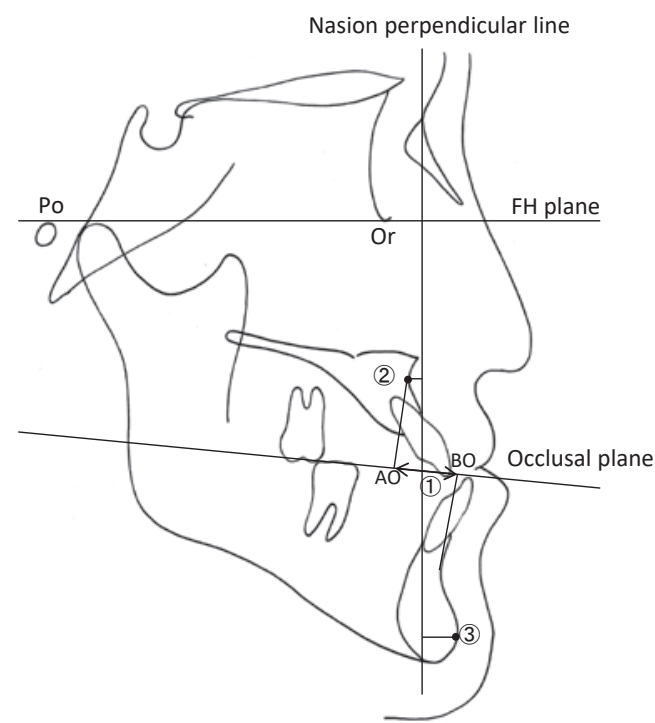

Fig. 2 Linear measurements used in cephalometric analysis

(1) Wits appraisal: distance from AO (point A perpendicular to occlusal plane) to $\mathrm{BO}$ (point $\mathrm{B}$ perpendicular to occlusal plane)

(2) Point A to nasion perpendicular (Pt-A): distance from point A to nasion perpendicular line horizontal to $\mathrm{FH}$ plane

(3) Pogonion to nasion perpendicular (Pog): distance from pogonion to nasion perpendicular line horizontal to $\mathrm{FH}$ plane

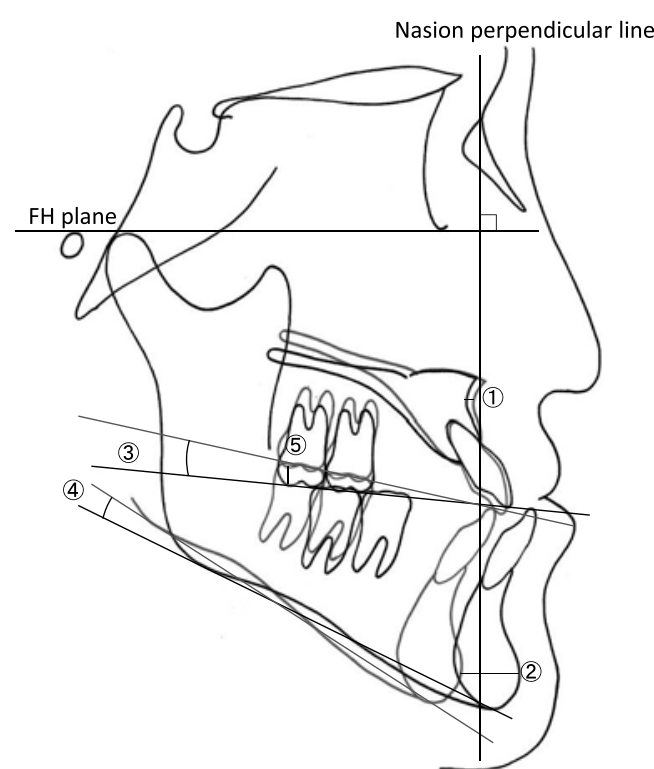

Fig. 3 Measurements used to evaluate surgical changes

(1) Amount of Anterior maxillary movement: difference between T0 Pt-A and T1 Pt-A

(2) Amount of mandibular setback: difference between T0 Pog and T1 Pog

(3) Amount of maxillary rotation: angular difference between $\mathrm{T} 0 \mathrm{OP}$ and $\mathrm{T} 1 \mathrm{OP}$

(4) Amount of mandibular rotation: angular difference between T0 MP and T1 MP

(5) Amount of posterior maxillary impaction: difference between $\mathrm{T} 0$ and $\mathrm{T} 1$ distance from $\mathrm{FH}$ plane to distal cusp tip of upper second molar.

Posterior maxillary impaction was calculated using difference in distance between molar cusps as palatal plane characteristically remains unchanged in HS.

involved Wits appraisal and those from the nasion perpendicular to Point A (Pt-A) and pogonion (Pog) respectively, as shown in Fig. 2.

(2) Evaluation of surgical changes

The measurements used to evaluate surgical changes are shown in Fig. 3. The amount of anterior maxillary movement was evaluated based on the difference between T0 Pt-A and T1 Pt-A. Mandibular setback was defined as the amount of the difference between T0 Pog and T1 Pog. Assessment of maxillary and mandibular rotation was performed using the angular difference between T0 OP and T1 OP, and T0 MP and T1 MP, respectively. Due 
to the characteristics of the HS procedure, the posterior nasal spine does not indicate the amount of posterior maxillary impaction; therefore, it was defined as the difference in vertical distance from the Frankfort plane to the distal cusp tip of the upper second molar between $\mathrm{T} 0$ and $\mathrm{T} 1$ in the HS group.

2) Measurements of morphology using CT data

Somatom Plus4 VolumeZoom (Siemens, Erlangen, Germany) was used for CT imaging of the maxillofacial region. The parameters adopted for imaging were as follows: tube voltage, $120 \mathrm{KV}$; tube current, $100 \mathrm{~mA}$; and FOV, $230 \mathrm{~mm}$. A built-in head fixation device was used in the CT scanner to enable more precise recreation of posture during scanning. Furthermore, to execute imaging under equal conditions, all patients were asked to swallow before commencing. Data with a voxel size of $0.45 \mathrm{~mm} \times 0.45 \mathrm{~mm} \times 1.25 \mathrm{~mm}$ were analyzed using Dolphin 3D software (Ver.11.7 Dolphin Imaging \& Management Solutions, California, USA) $)^{1,27}$. Reference planes were established to classify the upper airway into segmental regions upon evaluation of pharyngeal volume. Specifically, the Na plane (the plane perpendicular to the $\mathrm{FH}$ plane that passes the nasion); the $\mathrm{S}$ plane (the plane perpendicular to the $\mathrm{FH}$ plane that passes the most anterior point of the sphenoid sinus); the $\mathrm{CV}_{1}$ plane (the plane parallel to the $\mathrm{FH}$ plane that passes the most inferior point of the first cervical vertebra); the $\mathrm{CV}_{2}$ plane (the plane parallel to the $\mathrm{FH}$ plane that passes the most inferior point of the second cervical vertebra); and the $\mathrm{CV}_{3}$ plane (the plane parallel to the $\mathrm{FH}$ plane that passes the most inferior point of the third cervical vertebra) were established on multi-planar reconstruction (MPR) images. The airway was divided into 4 compartments using these reference planes, as shown in Fig. 4. The region from the Na plane to the $\mathrm{S}$ plane was defined as the nasal airway segment (NAS: A); that from the $S$ plane to the $\mathrm{CV}_{1}$ plane as the upper pharyngeal segment (UPS: B); that from the $\mathrm{CV}_{1}$ plane to the $\mathrm{CV}_{2}$ plane as the middle pharyngeal segment (MPS: C); and that from the
$\mathrm{CV}_{2}$ plane to the $\mathrm{CV}_{3}$ plane as the lower pharyngeal segment (LPS: D). The pharyngeal volume in each region was calculated.

3) Superimposition of pharyngeal morphology

Polygon data (STL format file) were used to enable 3D expression of the pharyngeal morphology at each time point (pre- and postoperatively) for a comparison. The construction process involved extracting the pharynx from the CT image data using Volume Extractor (Ver.3.0, i-Plants Systems, Iwate, Japan) and converting it into polygon data. Superimposition of each model was performed using SpGauge (2014.1. Armonicos Co., Ltd., Shizuoka, Japan). The best fit feature in SpGauge was used for superimposition and operated until minimal misalignment was obtained. After superimposition, the misaligned area between each image was colormapped to show the location and amount of change postoperatively, thus enabling visualization (Fig. 5).

4) Method of statistical analysis

The Shapiro-Wilk test revealed the data in this study to be non-parametric. The Wilcoxon signed-rank test was used to compare temporal changes in pharyngeal volume between T0 and T1. Furthermore, the MannWhitney $U$ test was used to evaluate the amount of change in pharyngeal volume, surgical change, and the maxillofacial values between T0 and T1 in the two groups. Spearman's rank correlation coefficient was adopted to assess the correlation between the amount of surgical change and the amount of change in pharyngeal volume in each region. The level of significance was set at $5 \%$. The SPSS Statistics software package (Ver.24.0, IBM, Armonk, NY, USA) was used for the statistical analysis.

\section{Results}

\section{Results of maxillofacial cephalometric analysis}

The results of the maxillofacial cephalometric analysis at $\mathrm{T} 0$ and $\mathrm{T} 1$ are shown in Tables 1 and 2, respectively. No significant 

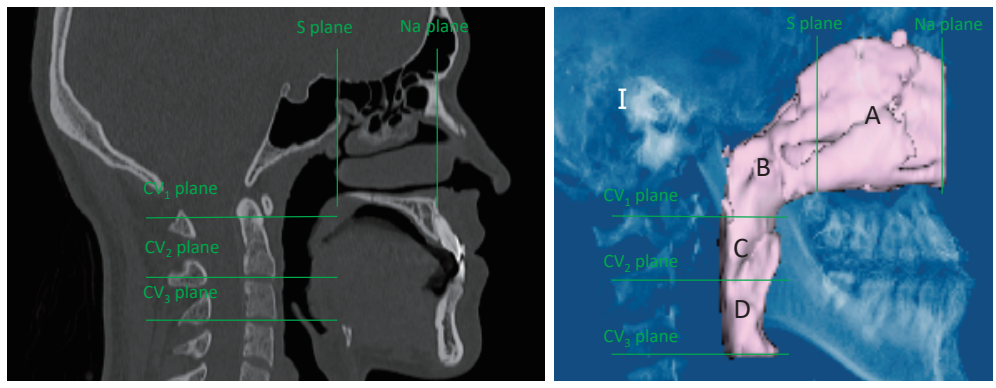

Fig. 4 Segmentation of upper airway

Airway was divided into 4 compartments by planes perpendicular and horizontal to $\mathrm{FH}$ plane.

A: Nasal airway segment (NAS), defined by Na plane and $\mathrm{S}$ plane

B: Upper pharyngeal segment (UPS), defined by $S$ plane and $\mathrm{CV}_{1}$ plane

C: Middle pharyngeal segment (MPS), defined by $\mathrm{CV}_{1}$ and $\mathrm{CV}_{2}$ plane

D: Lower pharyngeal segment (LPS), defined by $\mathrm{CV}_{2}$ and $\mathrm{CV}_{3}$ plane
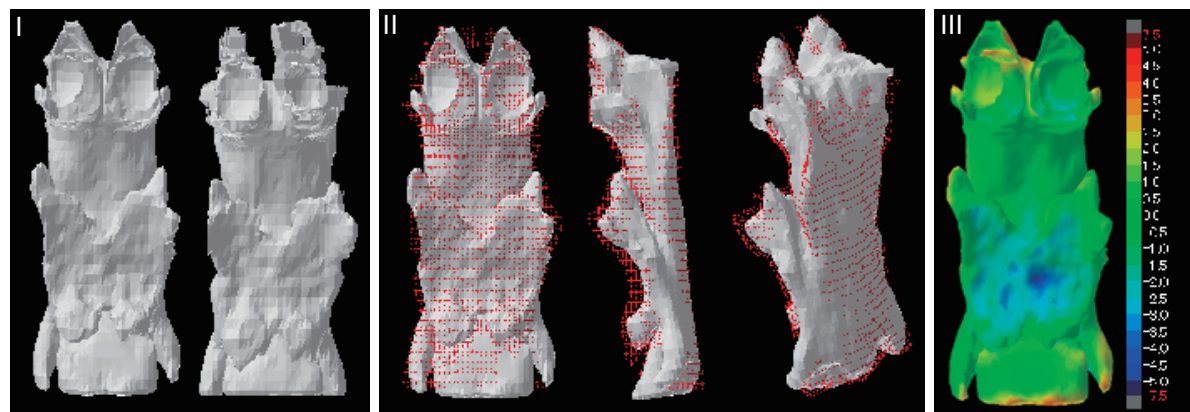

Fig. 5 Method of pharyngeal morphology superimposition

I: Polygon data of pharynx at pre- and postoperatively

II: Superimposition of pharynx using SP Gauge best fit feature

III: Color-mapping revealed changes in pharynx. Red zones show areas of enlargement; green show no change; and blue show constriction.

difference was found in the maxillofacial angular measurements between the two groups upon evaluation of the maxillofacial morphology at T0. In distance measurements, the Wits appraisal revealed a significantly smaller value and the Pog a significantly larger value in the HS group than in the LF group. At T1, while a significantly smaller value was observed in the Wits appraisal in the HS group than in the LF group, the other values showed no significant difference.

The results for amount of maxilla rotation, posterior maxilla impaction, and mandibular setback all revealed a significantly larger value in the HS group than in the LF group, as shown in Fig. 6. Meanwhile, no significant difference was observed in anterior maxillary movement or mandibular rotation.

\section{Results of pharyngeal volume measurement}

The results of pharyngeal volume measurement in each group are shown in Fig. 7. No significant change was observed in the NAS or MPS segments in either group between T0 and T1. The volume in the NAS segment saw a slight increase in the HS group, although this was not significant. A significant decrease 
Yoshino $\mathrm{N}$ et al.

Table 1 Maxillofacial cephalometric analysis at T0

\begin{tabular}{lccccc}
\hline \hline & \multicolumn{2}{c}{ HS $(\mathrm{n}=20)$} & $\mathrm{LF}(\mathrm{n}=20)$ & \\
\cline { 2 - 6 } & Mean & SD & Mean & SD & $\mathrm{p}$ \\
\hline SNA $\left({ }^{\circ}\right)$ & 81.5 & 2.3 & 79.8 & 4 & 0.086 \\
SNB $\left(^{\circ}\right)$ & 86 & 2.8 & 83.3 & 4.2 & 0.108 \\
ANB $\left({ }^{\circ}\right)$ & -4.5 & 3.8 & -3.5 & 2.2 & 0.904 \\
Mandibular plane angle $\left(^{\circ}\right)$ & 32.4 & 7.4 & 30.9 & 5.7 & 0.62 \\
Occlusal plane angle $\left(^{\circ}\right)$ & 12.3 & 4.9 & 12.5 & 3.7 & 0.678 \\
Pt-A (mm) & -1.1 & 2.5 & -1.2 & 2.3 & 0.925 \\
Pogonion $(\mathrm{mm})$ & 9.2 & 3.1 & 4.2 & 2 & $0.000 * *$ \\
Wits $(\mathrm{mm})$ & -13.1 & 3.2 & -10.6 & 2.4 & $0.013 *$ \\
\hline
\end{tabular}

Significantly different according to Mann-Whitney $U$ test, $*: \mathrm{p}<0.05 \quad * *: \mathrm{p}<0.01$

Table 2 Maxillofacial cephalometric analysis at T1

\begin{tabular}{lccccc}
\hline \hline & \multicolumn{2}{c}{ HS $(\mathrm{n}=20)$} & LF $(\mathrm{n}=20)$ & \\
\cline { 2 - 6 } & Mean & SD & Mean & SD & $\mathrm{p}$ \\
\hline SNA $\left({ }^{\circ}\right)$ & 81.3 & 3.5 & 80.8 & 2.3 & 0.06 \\
SNB $\left({ }^{\circ}\right)$ & 79.2 & 2.8 & 79.9 & 4.2 & 0.052 \\
ANB $\left({ }^{\circ}\right)$ & 2 & 3.8 & 1 & 2.2 & 0.64 \\
Mandibular plane angle $\left(^{\circ}\right)$ & 35.1 & 6.3 & 34.1 & 5.1 & 0.478 \\
Occlusal plane angle $\left(^{\circ}\right)$ & 17.4 & 3.2 & 16.2 & 3.4 & 0.265 \\
Pt-A (mm) & -0.5 & 2.5 & -0.8 & 1.7 & 0.718 \\
Pogonion (mm) & -1.4 & 3.1 & -1.8 & 1.3 & 0.583 \\
Wits (mm) & -5.8 & 2.6 & -4 & 1.7 & $0.011 *$ \\
\hline
\end{tabular}

Significantly different according to Mann-Whitney $U$ test, $*: \mathrm{p}<0.05$

was noted in the UPS in the LF group, whereas none was observed in the HS group. A significant decrease was observed in the LPS in both groups. Figure 8 shows comparison of the amount of change in pharyngeal volume between the two groups. No significant difference was seen in the NAS, UPS, or MPS segments; the LPS segment showed a significantly smaller value in the HS group than in the LF group, however.

\section{Superimposition of pharyngeal morphology change}

Representative superimposition images from each group are shown in Fig. 9. In the HS group, frontal observation revealed con- striction in the LPS, particularly in the area around the dorsum of the tongue. In the LF group, constriction was seen around the soft palate for the UPS and around the base of tongue and epiglottis for the LPS. The lateral view in both groups revealed no change in the lateral pharyngeal wall. The dorsal view also showed no change in the posterior pharyngeal wall.

\section{Correlation}

The results of the correlation analysis in both groups are shown in Tables 3 and 4, respectively. The amount of mandibular setback and LPS volume showed a negative correlation in the HS group. Meanwhile, no 

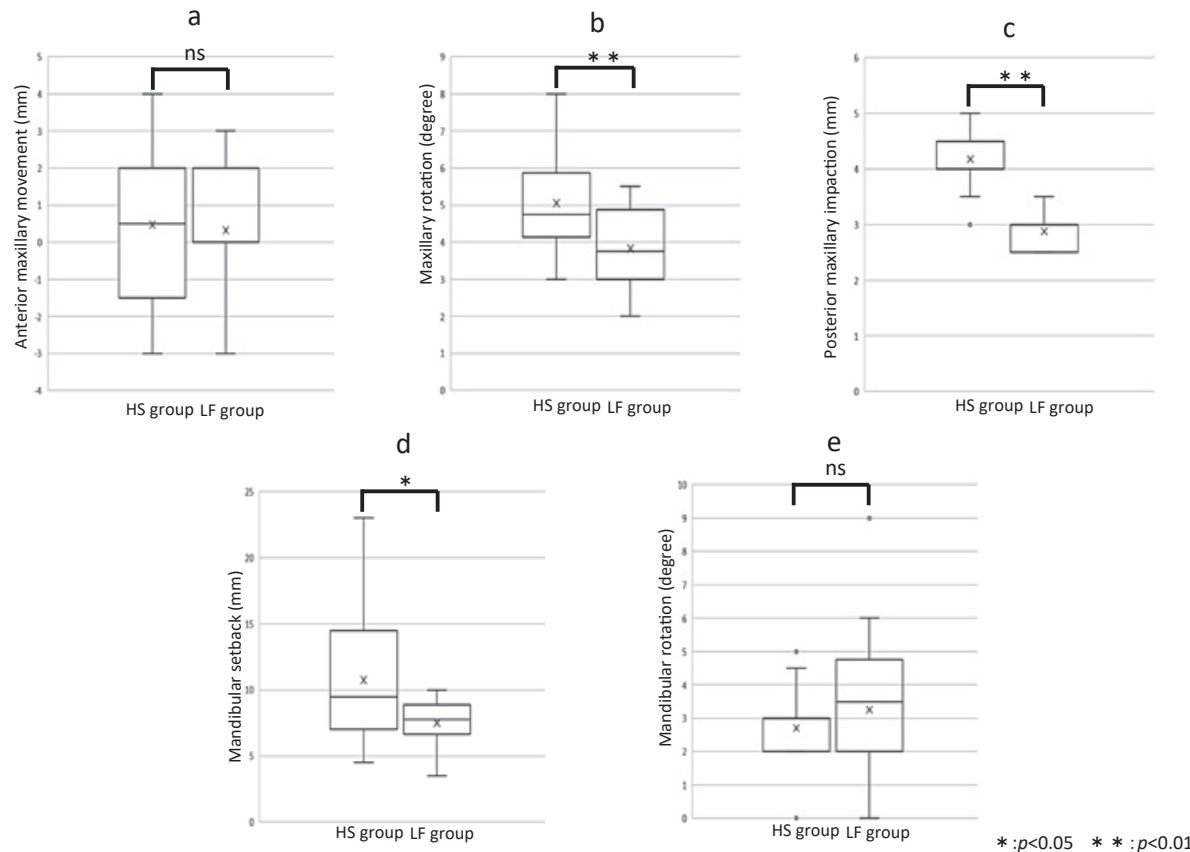

Fig. 6 Surgical changes observed between T0 and T1

Comparisons between HS and LF groups in amount of a: Anterior maxillary movement, b: Maxillary rotation, c: Posterior maxillary impaction, d: Mandibular setback, e: Mandibular rotation. Note significant difference in amount of maxillary rotation, posterior maxillary impaction, and mandibular setback.

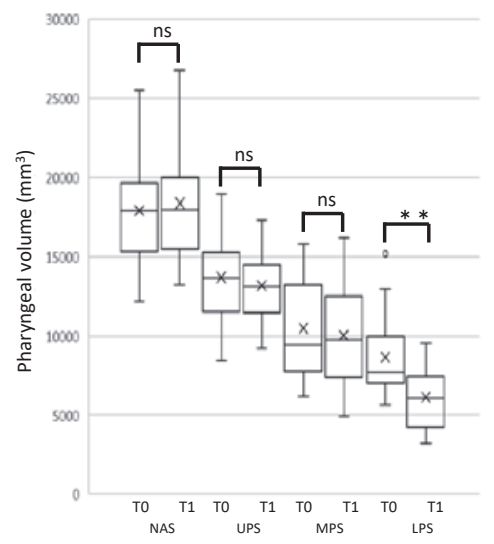

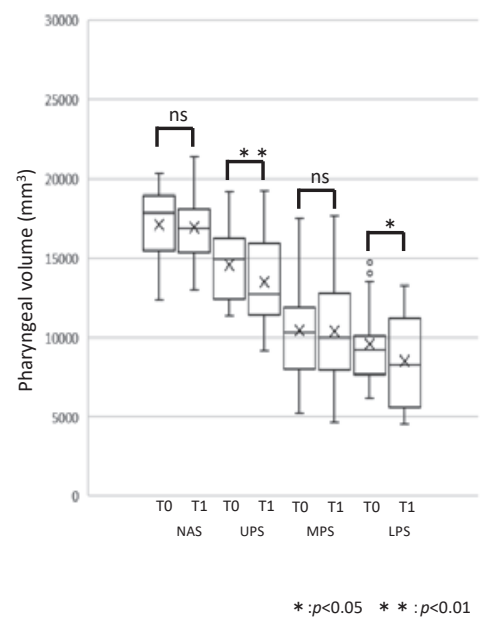

Fig. 7 Pharyngeal volume changes between T0 and T1

a: Changes in HS group, b: Changes in LF group

LPS volume showed significant decrease in both groups, whereas UPS showed significant decrease in LF group only. 


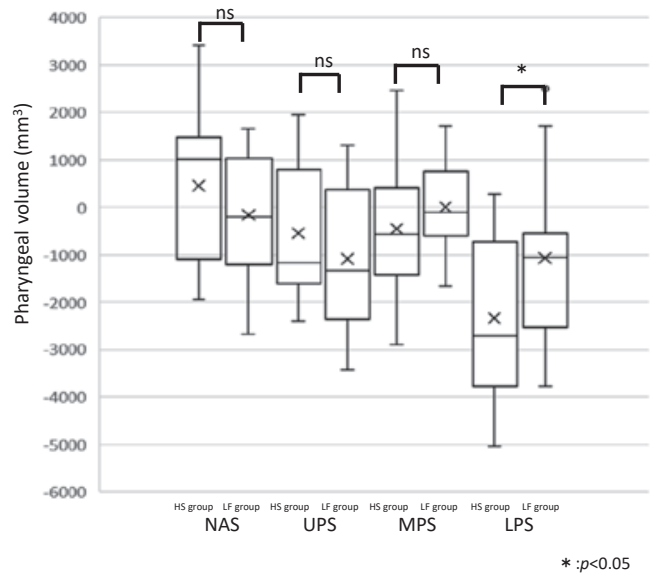

Fig. 8 Comparison of pharyngeal volume change between groups

NAS, UPS, and MPS showed no significant difference between HS and LF groups; LPS was significantly smaller in HS group. correlation was observed between the amount of posterior maxilla impaction and UPS volume. In the LF group, a negative correlation was found between the amount of posterior maxilla impaction and UPS volume. Negative correlations were found between LPS volume and the amount of maxillary rotation, and between LPS volume and amount of mandibular setback.

\section{Discussion}

A number of earlier studies have investigated the correlation between different methods of surgical movement and change in pharyngeal volume following orthognathic surgery in skeletal Class III cases ${ }^{2,4,6,10,11,13,15,19,20,22)}$. In some cases of mandibular prognathism,
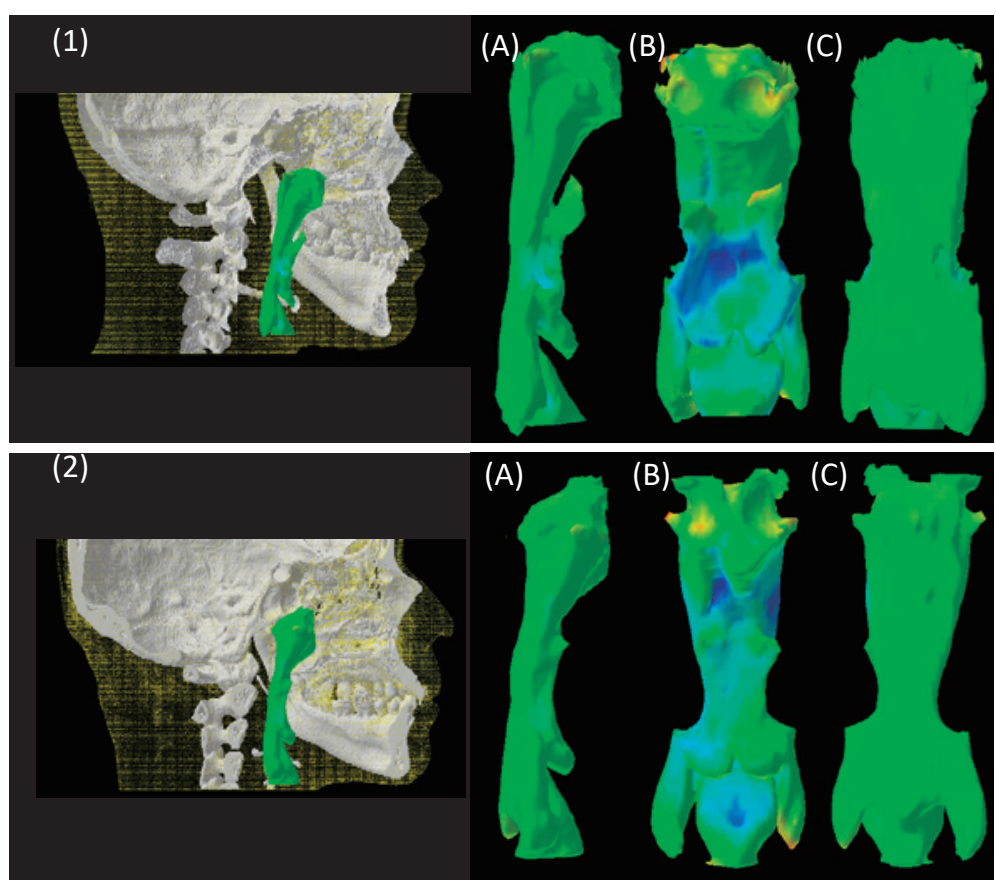

Fig. 9 Superimposition images of pharynx in HS (1) and LF groups (2)

Representative display is shown from (A) lateral, (B) frontal, and (C) posterior view. Green areas shows parts that remained relatively unchanged; constricted areas are shown in blue. 
Table 3 Spearman correlation (r) analysis between surgical changes and pharyngeal volume changes in HS group

\begin{tabular}{|c|c|c|c|c|c|c|c|c|c|c|}
\hline & \multicolumn{2}{|c|}{$\begin{array}{c}\text { Anterior } \\
\text { maxillary } \\
\text { movement }\end{array}$} & \multicolumn{2}{|c|}{$\begin{array}{c}\text { Amount of } \\
\text { posterior maxillary } \\
\text { impaction }\end{array}$} & \multicolumn{2}{|c|}{$\begin{array}{l}\text { Maxillary } \\
\text { rotation }\end{array}$} & \multicolumn{2}{|c|}{$\begin{array}{l}\text { Amount of } \\
\text { mandibular } \\
\text { setback }\end{array}$} & \multicolumn{2}{|c|}{$\begin{array}{l}\text { Mandibular } \\
\text { rotation }\end{array}$} \\
\hline & $\mathrm{r}$ & $\mathrm{p}$ & $\mathrm{r}$ & $\mathrm{p}$ & $r$ & $\mathrm{p}$ & $\mathrm{r}$ & $\mathrm{p}$ & $\mathrm{r}$ & $\mathrm{p}$ \\
\hline NAS & 0.255 & 0.278 & -0.351 & 0.130 & -0.073 & 0.760 & 0.085 & 0.722 & 0.201 & 0.395 \\
\hline UPS & 0.248 & 0.291 & 0.287 & 0.221 & -0.078 & 0.744 & 0.33 & 0.155 & 0.094 & 0.693 \\
\hline MPS & -0.306 & 0.199 & -0.236 & 0.318 & 0.042 & 0.862 & -0.146 & 0.538 & -0.097 & 0.683 \\
\hline LPS & -0.131 & 0.581 & -0.317 & 0.173 & -0.38 & 0.098 & -0.487 & $0.029 *$ & -0.271 & 0.247 \\
\hline
\end{tabular}

$*: \mathrm{p}<0.05$

Table 4 Spearman correlation ( $\mathrm{r}$ ) analysis between surgical changes and pharyngeal volume changes in LF group

\begin{tabular}{|c|c|c|c|c|c|c|c|c|c|c|}
\hline & \multicolumn{2}{|c|}{$\begin{array}{l}\text { Anterior } \\
\text { maxillary } \\
\text { movement }\end{array}$} & \multicolumn{2}{|c|}{$\begin{array}{c}\text { Amount of } \\
\text { posterior maxillary } \\
\text { impaction }\end{array}$} & \multicolumn{2}{|c|}{$\begin{array}{l}\text { Maxillary } \\
\text { rotation }\end{array}$} & \multicolumn{2}{|c|}{$\begin{array}{l}\text { Amount of } \\
\text { mandibular } \\
\text { setback }\end{array}$} & \multicolumn{2}{|c|}{$\begin{array}{l}\text { Mandibular } \\
\text { rotation }\end{array}$} \\
\hline & $\mathrm{r}$ & $\mathrm{p}$ & $\mathrm{r}$ & $\mathrm{p}$ & $r$ & $\mathrm{p}$ & $\mathrm{r}$ & $\mathrm{p}$ & $\mathrm{r}$ & $\mathrm{p}$ \\
\hline NAS & -0.021 & 0.930 & 0.194 & 0.413 & -0.061 & 0.797 & 0.329 & 0.157 & -0.06 & 0.802 \\
\hline UPS & -0.334 & 0.150 & -0.554 & $0.011 *$ & -0.362 & 0.117 & 0.171 & 0.471 & -0.027 & 0.911 \\
\hline MPS & 0.105 & 0.659 & 0.308 & 0.186 & 0.334 & 0.150 & 0.064 & 0.788 & -0.229 & 0.332 \\
\hline LPS & -0.298 & 0.167 & -0.383 & 0.095 & -0.513 & $0.022 *$ & -0.407 & $0.031 *$ & 0.061 & 0.798 \\
\hline
\end{tabular}

$*: \mathrm{p}<0.05$

the posterior maxilla is impacted so that the occlusal plane will rotate in a clockwise direction, which enables a larger amount of mandibular setback. Such movements, when executed with LF, however, have been reported to cause constriction in the nasopharynx, the oropharynx, and the hypopharyn $x^{11,15)}$. Some studies have noted the development of sleepdisordered breathing after mandibular setback $^{7,24,26)}$, although opinion remains divided on whether this affects sleep quality. The general consensus, however, is that a large amount of mandibular setback may induce respiratory disorder.

On the other hand, not many reports have been published on orthognathic surgery with HS and its effect on pharyngeal morphology. Harada was the pioneer in this respect, explaining its reliability in impaction of the posterior maxilla, as it separately mobilizes the maxilla into alveolar bone and palatal bone fragments after the down fracture seen in LF, thus evading interference in the posterior region. Other merits include a decrease in risk of damage to the descending palatine artery and reduced narrowing of the nasal cavity ${ }^{9}$. Kobayashi and Yoshioka followed by studying the amount of maxillary impaction and stability after orthognathic surgery performed with HS, confirming it to be as safe a procedure as $\mathrm{LF}^{17,28)}$. Furthermore, Kitamura concluded that HS was a dependable means of rotating the occlusal plane in a clockwise direction, making it invaluable in treating long-face cases of mandibular prognathism as it increases the amount of posterior-superior setback in the $\operatorname{chin}^{16)}$. As for reports on HS and pharyngeal morphology, Shimamine investigated the effect posterior-superior movement of the maxilla due to HS had on 
the airway in skeletal Class II cases and confirmed constriction in the oropharyngeal $\operatorname{area}^{25)}$. Since the above-mentioned studies were accomplished 2-dimensionally based on lateral cephalograms, 3D change remained unclear. Hence, the present study employed 3D evaluation to scrutinize the specific influence of HS on the airway and compare its effects with respect to this with those of LF. The number of cases involving HS orthognathic surgery, and the number where long-term postoperative observation was performed is limited, however. Therefore, cephalograms and CT data obtained at 1 month postoperatively were used here as postliminary records. In previous studies, assessment of the airway based on the results of magnetic resonance imaging revealed no signs of postoperative airway edema within 24 to 48 hours after orthognathic surgery ${ }^{21}$. This suggests that the present postoperative data allowed reliable assessment, without the need to take postoperative compromise into account.

In the present study, 3D analysis revealed constriction in the airway around the base of tongue in both groups, with airway volume in the LPS segment showing a significant decrease between $\mathrm{T} 0$ and $\mathrm{T} 1$. This, as reported in previous studies, may be due to the tongue and hyoid bone being thrust into a backwards position with the setback movement of the mandible $e^{4,14,20)}$. The average amount of mandibular setback in the HS group $(10.7 \mathrm{~mm})$ in the present study was greater than that in the LF group $(6.1 \mathrm{~mm})$. This may have resulted in the base and dorsum of the tongue exerting greater influence on the surrounding pharyngeal walls, which might explain the significantly larger decrease in LPS volume seen in the HS group. Although a comparison of the results for the UPS revealed no significant change in the HS group, a significant decrease was observed in volume in the AP in the LF group. Furthermore, a negative correlation was observed between the amount of posterior maxilla impaction and UPS volume in the LF group, while no correlation was observed in the HS group. This constriction seen around the soft palate in the LF group can be caused by impaction of the entire maxilla along with palatal bone that occurs in the LF procedure. In contrast, only the alveolar bone fragment is impacted with the HS procedure, and the palatal bone remains unmoved. This may explain the decreased influence on the soft palate and UPS observed in the HS group in the present study. Large changes in the vertical position of the nasal floor due to the distinctive surgical procedure adopted were anticipated in this study. Therefore, more reference planes were used than in the earlier study by Lee ${ }^{19)}$ to allow observation of its influence on nasal capacity. The results revealed that neither surgical method had any influence on the nasal airway. Any influence due to maxillary impaction may have been negated due to the large nasal capacity. Although a decrease in the volume of the nasal airway was anticipated following maxillary impaction, only a slight increase was observed in the HS group.

Evaluation of preoperative maxillofacial morphology revealed more pronounced mandibular prognathism in the HS group, with a larger anteroposterior discrepancy between the upper and lower jaw. Additionally, the amount of maxillary rotation, posterior maxillary impaction, and mandibular setback was significantly larger in the HS group than in the LF group. This suggests that there was greater impaction of the posterior maxilla in the HS group, so that the aim was to rotate the occlusal plane in a clockwise direction in order to achieve greater mandibular setback. This perspective is similar to that of Kitamura ${ }^{16)}$. Postoperative evaluation in the present study revealed no difference in maxillofacial morphology between the two groups. This demonstrates that HS offers similar results to LF in terms of postoperative facial esthetics in cases involving large skeletal discrepancies.

We believe that the present results are of importance for surgical planning in skeletal Class III cases. Recently, concerns have been expressed regarding the onset of OSA as a complication of orthognathic surgery ${ }^{8,24,26)}$. Surgical treatment plans must be made with 
caution when efforts to achieve large mandibular setbacks are involved. A fluid structure interaction study on OSA patients demonstrated an area of marked constriction and air resistance in the pharynx preoperatively; but when eliminated postoperatively, polysomnography outcomes greatly improved $^{5}$. Moreover, obstruction around not only the base of the tongue, but also the soft palate is indicated as an influencing factor in OSA $^{12,18,23)}$. Therefore, it is important not only to pay attention to the amount of mandibular setback, but also to select a surgical procedure that allows for minimal pharyngeal constriction around the soft palate and base of tongue in the planning of orthognathic surgery in patients at risk of airway constriction.

\section{Conclusion}

A 3D evaluation was conducted on pharyngeal morphology in skeletal Class III patients that underwent orthognathic surgery. A comparison between an LF and a HS group revealed postoperative morphological differences in the upper pharyngeal segment. The results demonstrated that HS had less effect on the nasopharynx, regardless of the amount of posterior maxilla impaction, possibly due to the non-movement of the palatal bone. We believe that the present results will prove valuable in making decisions involved in the planning of orthognathic surgery treatment.

\section{Acknowledgements}

All the authors of this article declare no conflict of interest with regards to the content of this study.

\section{References}

1) Alves MJ, Baratieri C, Mattos CT, Brunetto D, Fontes RC, Santos JRL, Ruellas ACO (2012) Is the airway volume being correctly analyzed?
Am J Orthod Dentofac Orthop 141:657-661.

2) Athanasiou AE, Toutountzakis N, Mavreas D, Ritzau M, Wenzel A (1991) Alterations of hyoid bone position and pharyngeal depth and their relationship after surgical correction of mandibular prognathism. Am J Orthod Dentofac Orthop 100:259-265.

3) Bailey LT, Proffit WR, White, RPJ (1995) Trends in surgical treatment of Class III skeletal relationships. Int $\mathrm{J}$ Adult Orthodon Orthognath Surg 10:108-118.

4) Brunetto DP, Velasco L, Koerich L, Araújo MTDS (2014) Prediction of 3-dimensional pharyngeal airway changes after orthognathic surgery: A preliminary study. Am J Orthod Dentofac Orthop 146:299-309.

5) Chang KK, Kim KB, McQuilling MW, Movahed R (2018) Fluid structure interaction simulations of the upper airway in obstructive sleep apnea patients before and after maxillomandibular advancement surgery. Am J Orthod Dentofac Orthop 153:895-904.

6) Chen F, Terada K, Hua Y, Saito I (2007) Effects of bimaxillary surgery and mandibular setback surgery on pharyngeal airway measurements in patients with Class III skeletal deformities. Am J Orthod Dentofac Orthop 131:372-377.

7) Fernández-Ferrer L, Montiel-Company JM, Pinho T, Almerich-Silla JM, Bellot-Arcís C (2015) Effects of mandibular setback surgery on upper airway dimensions and their influence on obstructive sleep apnoea - A systematic review. J Cranio-Maxillofacial Surg 43: 248-253.

8) Gandedkar NH, Chng CK, Por YC, Yeow VKL, Ow ATC, Seah TE (2017) Influence of bimaxillary surgery on pharyngeal airway in Class III deformities and effect on sleep apnea: A STOP-BANG Questionnaire and cone-beam computed tomography study.J Oral Maxillofac Surg 75:2411-2421.

9) Harada K, Ohkura K, Yonezawa H, Morishima S, Hirano Y, Enomoto S (2000) Postoperative stability of the maxilla after Le Fort I and horseshoe osteotomies in two-jaw surgery: Report of two cases. Nihon Kouku Geka Gakkai Zasshi 46:45-47. (in Japanese)

10) Hart PS, McIntyre BP, Kadioglu O, Currier GF, Sullivan SM, Li J, Shay C (2015) Postsurgical volumetric airway changes in 2-jaw orthognathic surgery patients. Am J Orthod Dentofac Orthop 147:536-546.

11) Hsieh YJ, Chen YC, Chen YA, Liao YF, Chen YR (2015) Effect of bimaxillary rotational setback surgery on upper airway structure in skeletal class III deformities. Plast Reconstr Surg 135: 361e-369e.

12) Isono S, Remmers JE, Tanaka A, Sho Y, Sato J, 
Nishino T (1997) Anatomy of pharynx in patients with obstructive sleep apnea and in normal subjects. J Appl Physiol 82:1319-1326.

13) Jakobsone G, Neimane L, Krumina G (2010) Two- and three-dimensional evaluation of the upper airway after bimaxillary correction of Class III malocclusion. Oral Surg, Oral Med Oral Pathol Oral Radiol Endodontol 110: 234-242.

14) Kawakami M, Yamamoto K, Fujimoto M, Ohgi K, Inoue M, Kirita T (2005) Changes in tongue and hyoid positions, and posterior airway space following mandibular setback surgery. J Craniomaxillofac Surg 33:107-110.

15) Kim MA, Kim BR, Choi JY, Youn JK, Kim YJR, Park YH (2013) Three-dimensional changes of the hyoid bone and airway volumes related to its relationship with horizontal anatomic planes after bimaxillary surgery in skeletal Class III patients. Angle Orthod 83:623-629.

16) Kitamura R, Kawamoto T, Miyamoto J, Kabasawa Y, Omura K, Kurohara K, Amagasa T, Moriyama K (2010) Dento-facial changes following a combination of Le Fort I with Horseshoe Osteotomy and a Bilateral Sagittal Spit Ramus Osteotomy for mandibular prognathism. Nihon Gakuhenkeisho Gakkai Zasshi 20:211-219. (in Japanese)

17) Kobayashi H, Harada K, Kikuchi T, Sato M, Morishima S, Kabasawa Y, Maruoka Y, Omura K (2004) Reliability of superior repositioning and postoperative stability of the maxilla treated with a combination of Le Fort I and Horseshoe Osteotomy. Nihon Gakuhenkeisho Gakkai Zasshi 14:43-48. (in Japanese)

18) Kyung SH, Park YC, Pae EK (2005) Obstructive sleep apnea patients with the oral appliance experience pharyngeal size and shape changes in three dimensions. Angle Orthod 75:15-22.

19) Lee Y, Chun YS, Kang N, Kim M (2012) Volumetric changes in the upper airway after bimaxillary surgery for skeletal Class III malocclusions: A case series study using 3-dimensional cone-beam computed tomography. J Oral Maxillofac Surg 70:2867-2875.

20) Li YM, Liu JL, Zhao JL, Dai J, Wang L, Chen JW (2014) Morphological changes in the pharyngeal airway of female skeletal Class III patients following bimaxillary surgery: A cone beam computed tomography evaluation. Int J Oral Maxillofac Surg 43:862-867.

21) Meisami T, Musa M, Keller MA, Cooper R, Clokie MLC, Sàndor GKB (2007) Magnetic resonance imaging assessment of airway status after orthognathic surgery. Oral Surg, Oral Med Oral Pathol Oral Radiol Endodontol 103: 458-463.

22) Park SB, Kim YI, Son WS, Hwang DS, Cho BH (2012) Cone-beam computed tomography evaluation of short- and long-term airway change and stability after orthognathic surgery in patients with Class III skeletal deformities: Bimaxillary surgery and mandibular setback surgery. Int J Oral Maxillofac Surg 41:87-93.

23) Pepin JL, Ferretti G, Veale D, Romand P, Coulomb M, Branbilla C (1992) Somnofluoroscopy, computed tomography, and cephalometry in the assessment of the airway in obstructive sleep apnoea. Thorax 47: 150-156.

24) Riley RW, Powell NB, Guilleminault C, Ware W (1987) Obstructive sleep apnea syndrome following surgery for mandibular prognathism. J Oral Maxillofac Surg 45:450-452.

25) Shimamine T, Yonemitsu I, Shibutani N, Koketsu M, Imai H, Fujita K, Omura S, Ono T (2018) Changes in upper airway morphology after Le Fort I and Horse-shoe Osteotomies in maxillary protrusion. Nihon Gakuhenkeisho Gakkai Zasshi 28:226-234. (in Japanese)

26) Uesugi T, Kobayashi T, Hasebe D, Tanaka R, Ike M, Saito C (2014) Effects of orthognathic surgery on pharyngeal airway and respiratory function during sleep in patients with mandibular prognathism. Int J Oral Maxillofac Surg 43:1082-1090.

27) Weissheimer A, Menezes LM, Sameshima, GT, Enciso R, Pham J, Grauer D (2012) Imaging software accuracy for 3-dimensional analysis of the upper airway. Am J Orthod Dentofac Orthop 142:801-813.

28) Yoshioka I, Khanal A, Kodama M, Habu M, Nishikawa T, Iwanaga K, Kokuryo S, Basugi A, Sakoda S, Fukuda J, Tominaga K (2011) A novel modification in combined Le Fort I and horseshoe osteotomy for posterior repostitioning of the maxilla. Asian J Oral Maxillofac Surg 23:172-176.

Correspondence:

Dr. Naoyuki Yoshino

Department of Orthodontics,

Tokyo Dental College,

1-2-2 Masago, Mihama-ku, Chiba

261-8502, Japan

E-mail: ny@yoc1988.com 